\title{
UAS-based imagery and photogrammetric processing for tree height and crown diameter extraction
}

\author{
Luís Pádua \\ University of Trás-os-Montes e Alto \\ Douro \\ Quinta de Prados, \\ 5001-801 Vila Real \\ +351259350356 \\ luispadua@utad.pt
}

\author{
Pedro Marques \\ University of Trás-os-Montes e Alto \\ Douro \\ Quinta de Prados, \\ 5001-801 Vila Real \\ +351259350 356 \\ pedro.marques@utad.pt
}

\author{
Telmo Adão \\ INESC Technology and Science \\ and University of Trás-os-Montes e \\ Alto Douro \\ Quinta de Prados, \\ 5001-801 Vila Real \\ +351259350356 \\ telmoadao@utad.pt
}

\author{
Raul Morais \\ INESC Technology and Science \\ and University of Trás-os-Montes e \\ Alto Douro \\ Quinta de Prados, \\ 5001-801 Vila Real \\ +351259350 356 \\ rmorais@utad.pt
}

\author{
António Sousa \\ INESC Technology and Science \\ and University of Trás-os-Montes e \\ Alto Douro \\ Quinta de Prados, \\ 5001-801 Vila Real \\ +351259350356 \\ amrs@utad.pt
}

\author{
Joaquim J. Sousa \\ INESC Technology and Science \\ and University of Trás-os-Montes e \\ Alto Douro \\ Quinta de Prados, \\ 5001-801 Vila Real \\ +351259350356 \\ jjsousa@utad.pt
}

\begin{abstract}
Advances in Unmanned Aerial Systems (UAS) allowed them to become both flexible and cost-effective. When combined with computer vision data processing techniques they are a good way to obtain high-resolution imagery and 3D information. As such, UAS can be advantageous both for agriculture and forestry areas, where the need for data acquisition at specific times and within a specific time frame is crucial, enabling the extraction of several measurements from different crop types. In this study a low-cost UAS was used to survey an area mainly composed by chestnut trees (Castanea sativa Mill.). Flights were performed at different heights (ranging from 30 to $120 \mathrm{~m}$ ), in single and double grid

Permission to make digital or hard copies of all or part of this work for personal or classroom use is granted without fee provided that copies are not made or distributed for profit or commercial advantage and that copies bear this notice and the full citation on the first page. Copyrights for components of this work owned by others than ACM must be honored. Abstracting with credit is permitted. To copy otherwise, or republish, to post on servers or to redistribute to lists, requires prior specific permission and/or a fee. Request permissions from Permissions@acm.org.
\end{abstract}

ICGDA '18, April 20-22, 2018, Prague, Czech Republic

(C) 2018 Association for Computing Machinery. ACM ISBN 978-1-4503-6445-4/18/04 ..\$15.00

DOI: https://doi.org/10.1145/3220228.3220241 flight patterns, and photogrammetric processing was then applied. The obtained information consists of orthophoto mosaics and digital elevation models which enable the measurement of individual tree's parameters such as tree crown diameter and tree height. Results demonstrate that despite its lower spatial resolution, data from single grid flights carried out at higher heights provided more reliable results than data acquired at lower flight heights. Higher number of images acquired in double grid flights also improved the results. Overall, the obtained results are encouraging, presenting a $\mathrm{R}^{2}$ higher than 0.9 and an overall root mean square error of $44 \mathrm{~cm}$.

\section{CCS Concepts}

-Applied computing $\rightarrow$ Environmental sciences Information systems $\rightarrow$ Geographic information systems; Data analytics

\section{Keywords}

Remote sensing; unmanned aerial systems; forestry; canopy height models; photogrammetric processing; chestnut trees

\section{INTRODUCTION}

Tree height and tree crown diameter determination are important operations due to both biological and commercial reasons [1]. When done using data acquired by remote sensing techniques it as proven to be an efficient and accurate way for forestry monitoring [2]. However, to have decision support systems for sustainable 
forest management practices, multi-temporal and accurate data is needed [3]. As such, Unmanned Aerial Systems (UAS) constitute a suitable mean to provide very high-resolution data with great flexibility, capable of carrying different sensors [4].

To survey forest areas, different sensors can be used depending on the task in hand. In the literature, several studies covering this subject can be found. A common used sensor is Light Detection And Ranging (LiDAR), which can accurately obtain trees' information from digital elevation models (DEM) $[2,5,6]$. Hyperspectral sensors can also enable trees' classification and monitor their health [7, 8]. However, approaches with low-cost RGB sensors combined with photogrammetric processing emerge as a valid alternative to those expensive sensors [9]. Indeed, Gatziolis et al. [3] explored the use of small Unmanned Aerial Vehicles (UAV) to obtain information from trees or small groups of trees. Other authors provided methods towards an automatic trees' parameters extraction [1, 10-12].

However, the accuracy of the aforementioned operations can be affected by different factors, namely biological and technical factors (related to data acquisition or to data processing) [6].

This study addresses the impact of different data acquisition parameters in data processing and consequently in the final products, using a low-cost UAV equipped with a RGB sensor.

The document is structured as follows: next section describes the materials and the methods used. Section 3 presents the obtained results and respective discussion, while in section 4 conclusions and future directions towards new possibilities from the obtained results are presented.

\section{SYSTEM AND EXPERIMENT DESCRIPTION}

In this section the used UAS is described along with the studied area and the data processing of the acquired imagery.

\subsection{UAV platform}

A DJI Phantom 4 multirotor (DJI, Shenzhen, China) was used to acquire the data. It is a low-cost and flexible UAS, able to automatically perform flights in different configurations through a set of user-defined waypoints. This UAV is equipped with a rolling-shutter 1/2.3" CMOS sensor that can acquire RGB imagery with $12.4 \mathrm{MP}$ resolution, attached to a 3-axis electronic gimbal. Moreover, its $5350 \mathrm{mAh}$ capacity battery that can last around 28 minutes in flight operations and it has also a Global Navigation Satellite System (GNSS) receiver to geolocate the acquired images and to position the UAV. For this study, the Pix4Dcapture (Pix4D SA, Lausanne, Switzerland) application was used for flight planning and its execution. It enables the user to define an area to survey, to do so by using single or double-grid flights and to set parameters as flight height, camera angle, UAV speed and the overlap percentage between the images.

\subsection{Study area}

The UAV flights were carried out in an area of approximately $5000 \mathrm{~m}^{2}$, mainly covered by chestnut trees (Castanea Sativa Mill.), located in a rural area $\left(41^{\circ} 22^{\prime} 42.7^{\prime \prime} \mathrm{N} 7^{\circ} 35^{\prime} 01.6^{\prime \prime} \mathrm{W}\right)$ in the north of Portugal. On June 4, 2017, at 2:30pm GMT, seven flights were conducted at different heights above ground level. It took approximately 25 minutes to complete all the flights. The planned flights covered a 100 by $100 \mathrm{~m}$ area with $70 \%$ overlap between images (longitudinal and lateral), with a camera angle set to $80^{\circ}$. These parameters were chosen to achieve accurate photogrammetric outcomes and to obtain 3D information for a better characterization of objects above ground level. The surveyed area was constituted by trees with different heights, crown diameters and also had connected tree canopies with no visible area to the soil. A total of 55 trees were analyzed and their crown diameter were measured in the field. Figure 1 presents an overview of the surveyed field, along with the covered area by UAV flights and the analyzed trees.

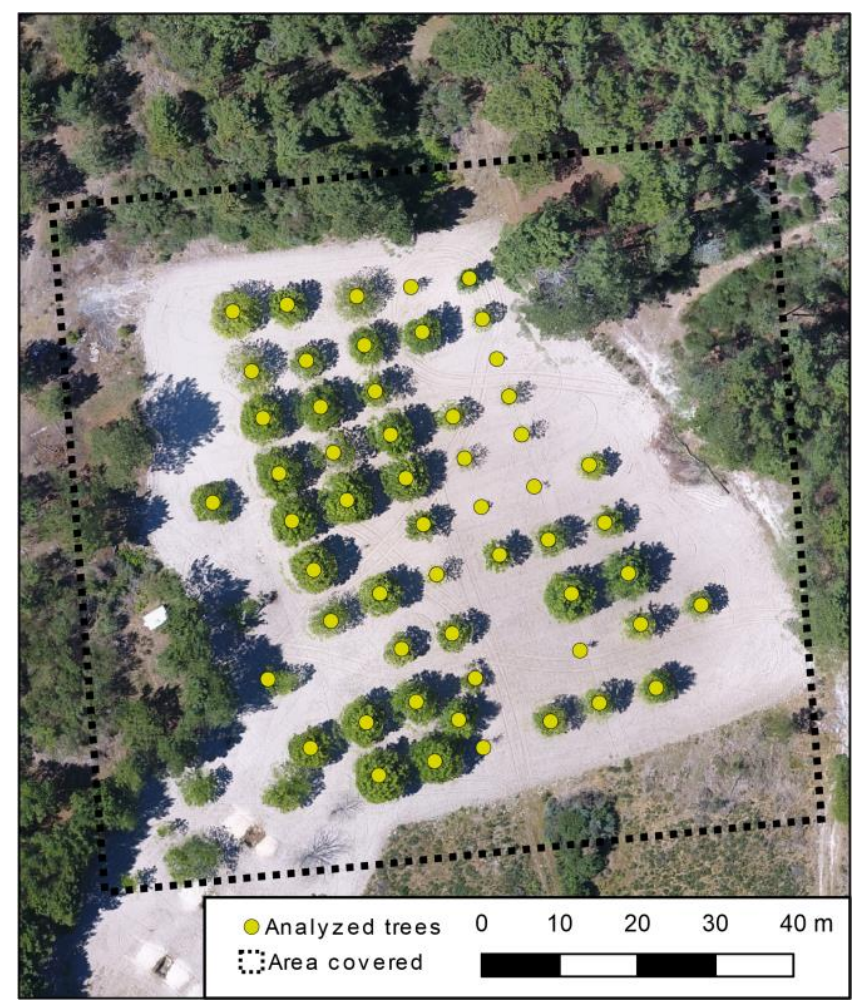

Figure 1 Overview of the surveyed area and analyzed trees.

Since the surveyed area were surrounded by pine (Pinus Pinaster) trees, the minimum flight height was set to $30 \mathrm{~m}$ to prevent any possible damages to the UAV and the maximum flight height was of $120 \mathrm{~m}$, which is the top flight height allowed in Portugal, according to the current legislation [13]. Carrying out flights above this limit would require an authorization from the Portuguese Civil Aviation Authority. Other flights were at 50 and $60 \mathrm{~m}$ height above ground level, following a single-grid pattern. Flights in a double grid (DG) pattern were also carried out, at 50, 60 and $120 \mathrm{~m}$ heights, with the goal to study its influence when comparing to single-grid flights. The number of images acquired and their ground sample distance (GSD) are provided in Table 1.

Table 1 Number of acquired images per flight and their corresponding average GSD.

\begin{tabular}{|c|c|c|}
\hline Flight height $(\mathbf{m})$ & Number of images & GSD $(\mathbf{c m})$ \\
\hline 30 & 102 & 1.31 \\
\hline 50 & 60 & 2.16 \\
\hline $50 \mathrm{DG}$ & 118 & 2.14 \\
\hline 60 & 44 & 2.65 \\
\hline $60 \mathrm{DG}$ & 89 & 2.64 \\
\hline 120 & 21 & 5.22 \\
\hline $120 \mathrm{DG}$ & 46 & 5.25 \\
\hline
\end{tabular}




\subsection{Data processing}

The photogrammetric software Pix4Dmapper Pro (Pix4D SA, Lausanne, Switzerland), was used to process the acquired imagery to generate dense point clouds. This software can provide different outcomes, valuable for geodetic analysis, and deal with possible distortions from rolling-shutter sensors, as the one used in this study. The acquired images were used to create multiple projects, corresponding to each performed flight. Pix4Dmapper processing pipeline is divided into three main steps: (1) Initial processing, responsible for detecting matching points (tie points) from the provided imagery. It also optimizes the location of the estimated features according to the camera's location and orientation in each image and its internal parameters. Then, a sparse point cloud is generated; (2) Point cloud and Mesh, where the obtained sparse point cloud is used to create a dense point cloud, which in turn enables the creation of a 3D textured mesh; and (3) DSM, Orthomosaic and Index responsible for the computation of different outcomes as Digital Surface Model (DSM), orthophoto mosaics, contour lines and Digital Terrain Model (DTM). There is also the possibility to compute vegetation indices with arithmetic operations from different imagery bands.

Within this study's scope data was processed as follows: sparse point clouds were obtained by using full keypoints matching from the default image scale. The matching image pairs were optimized for aerial grid based in the images' capture time, triangulation between images' geolocation, among others. Dense point clouds were then computed from a half image size, with a high point density and a minimum of three matches per each estimated 3D point. A $3 \mathrm{D}$ textured mesh was not computed. When producing raster outputs (orthophoto mosaic, DSM and DTM), a noise filter was applied to the dense point cloud to remove outlier points and a sharp surface smoothing filter was applied. The DSM was generated by interpolating the dense point cloud using a triangulation algorithm based on Delauney triangulation, originally proposed in [14]. This method was used since it is recommended for flat areas such as agriculture fields and stockpiles [15]. Orthophoto mosaics were computed along with DTMs that were generated by filtering possible objects above ground level. Photogrammetric processing of the UAV-based data was performed in a workstation equipped with an Intel ${ }^{\circledR}$ Xeon ${ }^{\circledR}$ CPU E5-2680 v4@ @2.40GHz, 32GB of RAM, a NVIDIA ${ }^{\circledR}$ Quadro ${ }^{\circledR}$ M4000 GPU and 1 TB SDD storage. Figure 2 shows the detail of the computed orthophoto mosaics. At higher GSD values lower image detail is noticeable. However, improvements are visible from both flights at $50 \mathrm{~m}$ height

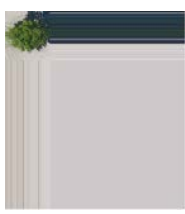

a)

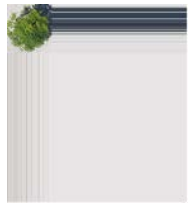

d)

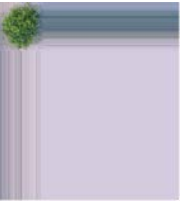

b)

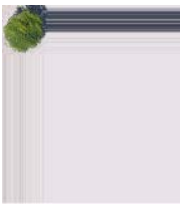

e)

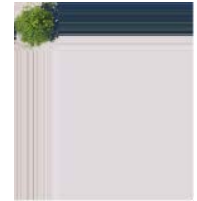

c)

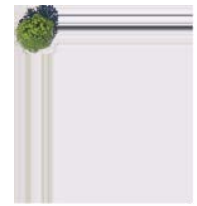

f)

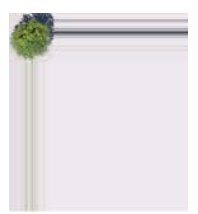

g)

Figure 2 Detail of the computed orthophoto mosaics for the same tree a) $30 \mathrm{~m}$, b) $50 \mathrm{~m}$ single-grid, c) $50 \mathrm{~m} \mathrm{DG}$, d) $60 \mathrm{~m}$ e) $60 \mathrm{~m}$ DG f) $120 \mathrm{~m}$ and g) $120 \mathrm{~m}$ DG.

Canopy Height Models (CHM) is a usual computed product for individual tree parameters estimation from aerial remote sensing data [16]. It is obtained by subtracting the generated DTM to the DSM, as presented in equation 1 . This is a reliable and fast way to obtain information about canopy height.

$$
\mathrm{CHM}=\mathrm{DSM}-\mathrm{DTM}
$$

The computation of such a model enables the extraction of information related to the objects above ground level. This is achieved replacing the altitude values of DSM and DTM by the differences among them. Values close to zero represent the ground and other values represent objects present in the surface. A visual representation of the CHM in comparison to DSM and DTM of the surveyed area is show in Figure 3.

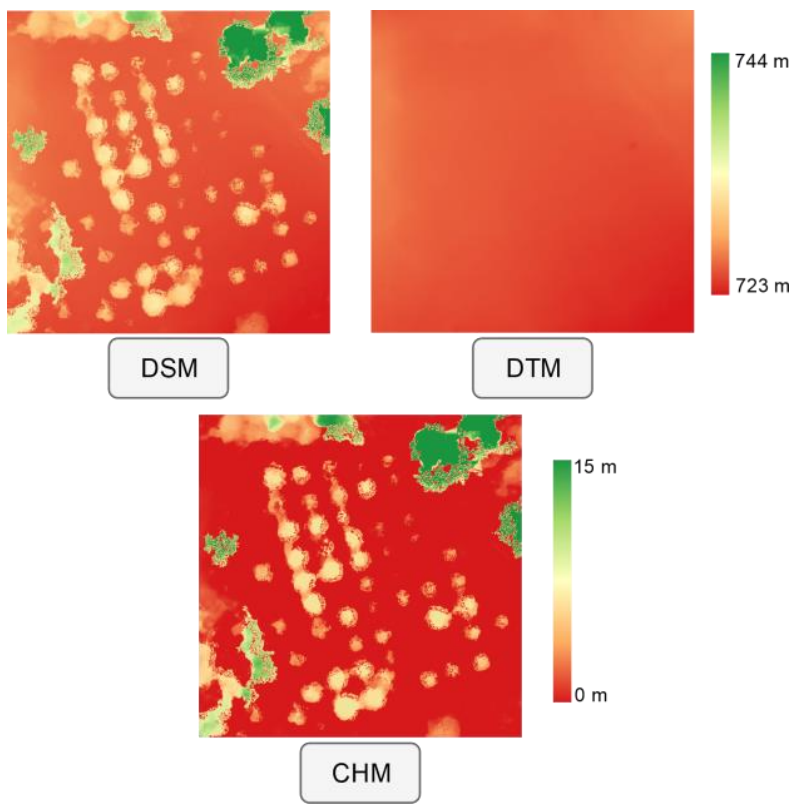

Figure 3 Color coded representations of DSM, DTM and CHM of the surveyed area from data acquired at $120 \mathrm{~m}$ height.

\section{RESULTS AND DISCUSSION}

The computed orthophoto mosaics, DSMs and DTMs were imported to QGIS, a free and open source Geographic Information System (GIS), for CHM computation and extraction of trees' parameters from the produced data, namely each tree's height and crown diameter.

\subsection{Tree crown diameter estimation}

Tree crown diameter values were estimated by measuring each tree in QGIS and compared with the values measured in the field (both observed and estimated values were rounded to the nearest decimal value). Tree crown diameter values measured in the field 
ranged from 0.5 to $7.5 \mathrm{~m}$. Most of the trees had a crown diameter between 4.5 and $6.5 \mathrm{~m}$, representing approximately $52 \%$ of the analyzed trees, as presented in Figure 4.

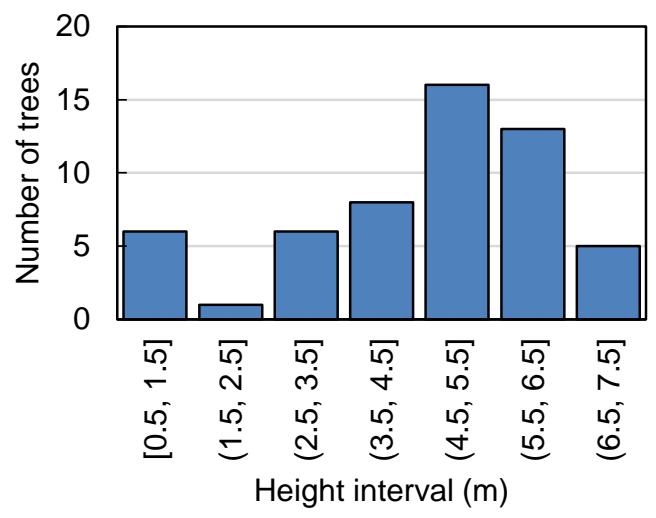

Figure 4 Tree crown diameter histogram representing values measured in the field.

The estimated crown diameter values were subjected to a statistical analysis, where different parameters were calculated, from the differences (D) of the values measured in the field (M) with the measured values in the orthophoto $(\mathrm{P})$, as defined in equation 2 .

$$
\mathrm{D}=\mathrm{P}-\mathrm{M}
$$

From this difference, the arithmetic mean $(\mu)$, standard deviation $(\sigma)$ and root mean square error (RMSE) were calculated for the outcomes correspondent to each flight height. The formulations used are shown in equations 3-6, where " $i$ " represents the number of each tree and " $n$ " the number of analyzed trees. Coefficient of determination $\left(\mathrm{R}^{2}\right)$ was also calculated from in-field measurements and the ones obtained in QGIS, as shown in equation 7.

$$
\begin{gathered}
\sigma=\sqrt{\frac{\sum_{i=1}^{n}\left|D_{i}-\mu\right|^{2}}{n}} \\
\mu=\frac{1}{n} \sum_{i=1}^{n} D_{i} \\
\text { RMSE }=\frac{1}{n} \sqrt{\sum_{i=1}^{n}\left(D_{i}\right)^{2}} \\
R^{2}=\sum_{i=1}^{n}\left(D_{i}-\mu\right)^{2}
\end{gathered}
$$

The results of tree crow diameter estimation are presented in Table 2. As observed, the data driven from all flights showed a $\mathrm{R}^{2}$ bigger than 0.9 , reaching 0.96 on DG flights, followed by the single grid flights with 0.95 at $120 \mathrm{~m}, 50$ and $60 \mathrm{~m}$ with 0.93 and $30 \mathrm{~m}$ with 0.91 . RMSE values confirm the same tendency as $\mathrm{R}^{2}$, with the values deviating $\pm 0.36 \mathrm{~m}$ for $50 \mathrm{DG}$ flight data and \pm 0.37 for the other two DG flights. This deviation value increases up to $\pm 0.60 \mathrm{~m}$ for the $30 \mathrm{~m}$ flight data. Standard deviation values also follow the same tendency as RMSE, as the values range from \pm 0.33 (60 m DG) $\mathrm{m}$ to $0.58 \mathrm{~m}$ ( $30 \mathrm{~m}$ single grid).
Therefore, the less favorable results were obtained from data collected in the lower height flight $(30 \mathrm{~m})$, despite its higher GSD (cm/pixel). Flights carried out in a single grid pattern show improvements when the height increases. On the other hand, when directly comparing single grid with DG flights, there is clear improvement at all flights heights, this is due to the number of images (almost double), being the results almost in the same line, independently from the flight height.

Table 2 Results from tree crown diameter estimation for the evaluated trees.

\begin{tabular}{|c|c|c|c|c|}
\hline Flight height (m) & $\mathbf{R}^{\mathbf{2}}$ & $\boldsymbol{\mu}$ & $\boldsymbol{\sigma}$ & $\mathbf{R M S E}$ \\
\hline 30 & 0.91 & -0.17 & 0.58 & 0.60 \\
\hline 50 & 0.93 & 0.11 & 0.46 & 0.47 \\
\hline $50 \mathrm{DG}$ & 0.96 & 0.12 & 0.34 & 0.36 \\
\hline 60 & 0.93 & 0.04 & 0.45 & 0.45 \\
\hline $60 \mathrm{DG}$ & 0.96 & 0.04 & 0.37 & 0.37 \\
\hline 120 & 0.95 & 0.16 & 0.40 & 0.43 \\
\hline $120 \mathrm{DG}$ & 0.96 & 0.17 & 0.33 & 0.37 \\
\hline
\end{tabular}

\subsection{Tree height estimation}

Tree height estimation was evaluated based on the computed CHM, since tree height information can easily be retrieved from this UAS-based outcome. Unlike tree crown diameter, tree heights were not measured in the field due to the lack of proper equipment. The evaluation of this parameter was mainly performed based on the presence of height information for each evaluated tree in the CHM. This way, three parameters where defined as: (1) good detection, the tree was totally detected in the CHM and the height can be easily retrieved from it; (2) undetected, as there is a clear absence of information about the tree height in the CHM; and (3) partial detection, as information about tree height exists, however it does not clearly correspond to the complete tree canopy. Results from this evaluation are presented in Figure 5.

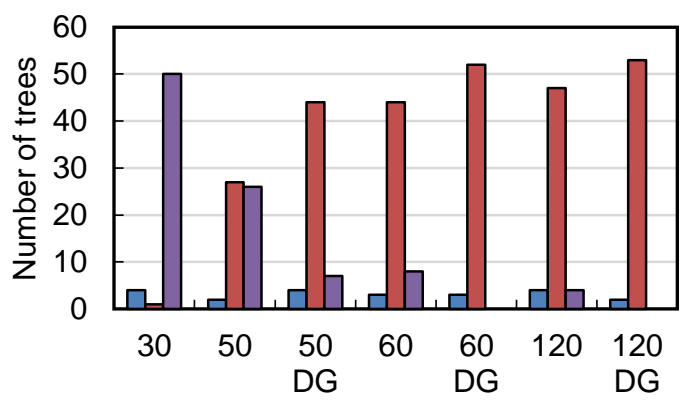

Flight height (m)

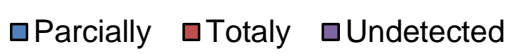

Figure 5 Detection results of the analyzed trees from the CHM of each flight.

The small number of detected trees in lower-height flights can be related to the perspective distortions caused by the high number of images. Being easier to detect visual similarities between overlapping images in vegetation areas when surveying at higher heights. In the other hand, trees reported as not being detected at $120 \mathrm{~m}$ flight were related to small-sized trees. However, the number of images influenced the CHM reliability, this is 
especially noticeable when comparing both flights at $50 \mathrm{~m}$ height above ground level. Despite the number of non-detected trees remain the same there was an increment of the number of good detection (from 27 to 44). DG flights at 120 and $60 \mathrm{~m}$ height had no undetected tree heights.

\section{CONCLUSIONS AND FUTURE WORK}

The amount of data generated from a UAV flight can be optimized. This is crucial when surveying big areas within a small-time frame and a good spatial resolution, saving time on batteries replacement or take-off and landing operations.

In this study, both tree crown diameter and tree height where evaluated for 55 trees from RGB imagery acquired at different flight heights above ground level. Results showed that the flight at the highest height $(120 \mathrm{~m})$ achieved the best overall results for both parameters in the single grid flight pattern. Moreover, improvements obtained in the DG flight showed the importance of performing this type of flights, when possible, to improve the results accuracy. However, a trade-off between sensor type, flight height, area to cover, amount of acquired data and UAV characteristics must be carefully analyzed depending on the task to perform.

Based on the obtained results, the same area kept being surveyed for multi-temporal analysis of chestnut trees. A DG flight pattern was selected and flights were carried out at two different heights (120 and $60 \mathrm{~m})$. This opened the possibility to combine imagery from different flight heights during photogrammetric processing. As future work, using this approach together with multi-temporal observations enables to monitor the presence of phytosanitary problems in earlier stages, and also to estimate harvesting seasons and production. More sources of data will provide the estimation of more parameters (e.g. vegetative vigor) using vegetation indices computed from multi-spectral data, water stress from thermal data and even to distinguish between tree species using hyperspectral data.

\section{ACKNOWLEDGMENTS}

This work was financed by the European Regional Development Fund (ERDF) through the Operational Programme for Competitiveness and Internationalisation - COMPETE 2020 under the PORTUGAL 2020 Partnership Agreement, and through the Portuguese National Innovation Agency (ANI) as a part of project "PARRA - Plataforma integrAda de monitoRização e avaliação da doença da flavescência douRada na vinhA" (No 3447) and ERDF and North 2020 - North Regional Operational Program, as part of project "INNOVINEandWINE - Vineyard and Wine Innovation Platform" (NORTE-01-0145-FEDER-000038).

\section{REFERENCES}

[1] Panagiotidis, D. et al. 2017. Determining tree height and crown diameter from high-resolution UAV imagery. International Journal of Remote Sensing. 38, 8-10 (May 2017), 2392-2410.
[2] Zhen, Z. et al. 2016. Trends in Automatic Individual Tree Crown Detection and Delineation-Evolution of LiDAR Data. Remote Sensing. 8, 4 (Apr. 2016), 333.

[3] Gatziolis, D. et al. 2015. 3D Tree Dimensionality Assessment Using Photogrammetry and Small Unmanned Aerial Vehicles. PLOS ONE. 10, 9 (set 2015), e0137765.

[4] Pádua, L. et al. 2017. UAS, sensors, and data processing in agroforestry: a review towards practical applications. International Journal of Remote Sensing. 38, 8-10 (May 2017), 2349-2391.

[5] Fassnacht, F.E. et al. 2016. Review of studies on tree species classification from remotely sensed data. Remote Sensing of Environment. 186, (Dec. 2016), 64-87.

[6] Stereńczak, K. 2013. Factors influencing individual tree crowns detection based on airborne laser scanning data. Forest Research Papers. 74, 4 (2013), 323-333.

[7] Näsi, R. et al. 2015. Using UAV-Based Photogrammetry and Hyperspectral Imaging for Mapping Bark Beetle Damage at Tree-Level. Remote Sensing. 7, 11 (Nov. 2015), 15467-15493.

[8] Nevalainen, O. et al. 2017. Individual Tree Detection and Classification with UAV-Based Photogrammetric Point Clouds and Hyperspectral Imaging. Remote Sensing. 9, 3 (Feb. 2017), 185.

[9] Zarco-Tejada, P.J. et al. 2014. Tree height quantification using very high resolution imagery acquired from an unmanned aerial vehicle (UAV) and automatic 3D photoreconstruction methods. European Journal of Agronomy. 55, (Apr. 2014), 89-99.

[10] Mohan, M. et al. 2017. Individual Tree Detection from Unmanned Aerial Vehicle (UAV) Derived Canopy Height Model in an Open Canopy Mixed Conifer Forest. Forests. 8, 9 (Sep. 2017), 340.

[11] Ok, A.O. and Ozdarici-Ok, A. 2017. 2-D delineation of individual citrus trees from UAV-based dense photogrammetric surface models. International Journal of Digital Earth. 0, 0 (Jun. 2017), 1-26.

[12] Surový, P. et al. 2018. Estimation of positions and heights from UAV-sensed imagery in tree plantations in agrosilvopastoral systems. International Journal of Remote Sensing. (Feb. 2018).

[13] 2016. Diário da República n. ${ }^{\circ}$ 238/2016, , Série II.

[14] Delaunay, B. 1934. Sur la sphere vide. Izv. Akad. Nauk SSSR, Otdelenie Matematicheskii i Estestvennyka Nauk. 7, 793-800 (1934), 1-2.

[15] Menu Process > Processing Options... > 3. DSM, Orthomosaic and Index > DSM and Orthomosaic: http://support.pix4d.com/hc/en-us/articles/202557769-

Menu-Process-Processing-Options-3-DSM-Orthomosaicand-Index-DSM-and-Orthomosaic. Accessed: 2018-02-10.

[16] Popescu, S.C. 2007. Estimating biomass of individual pine trees using airborne lidar. Biomass and Bioenergy. 31, 9 (Sep. 2007), 646-655. 